\title{
A NOTE ON A NUMBER THEORETICAL PAPER OF SIERPINSKI
}

\section{ALFRED BRAUER}

W. Sierpinski [5] has just published the following theorem:

"The set $A$ of all primes which are divisors of integers of form $2^{r}+1$ contains all primes of the form $8 n \pm 3$ and infinitely many primes of the form $8 n+1$. The set $B$ of all primes which are divisors of integers of the form $2^{2 s+1}-1$ contains all primes of the form $8 n+7$ and some primes of the form $8 n+1$. Every prime of form $8 n+1$ belongs either to $A$ or to $B$. The question whether the set $B$ contains infinitely many primes of form $8 n+1$ is raised, but remains open."

In this note a simple proof of this result will be given. Moreover, it will be shown that $B$ contains infinitely many primes of form $8 n+1$. More exactly, we prove a little more.

TheOREM 1. Let a be a given positive integer. An odd prime $p$ is a divisor of an integer of form $a^{r}+1$ if and only if a belongs to an even exponent $\bmod p$. The odd prime $q$ is a divisor of an integer of form $a^{28+1}-1$ if and only if $a$ belongs to an odd exponent $\bmod q$.

Proof. If $a$ belongs to an even exponent $2 k(\bmod p)$, then

$$
a^{2 k} \equiv 1(\bmod p),
$$

hence

$$
\begin{aligned}
\left(a^{k}+1\right)\left(a^{k}-1\right) & \equiv 0(\bmod p), \\
a^{k}+1 & \equiv 0(\bmod p)
\end{aligned}
$$

since otherwise $2 k$ would not be the exponent to which $a$ belongs $(\bmod p)$. Conversely, if $p$ divides $a^{r}+1$, then

$$
\begin{aligned}
a^{r} & \equiv-1(\bmod p), \\
a^{2 r} & \equiv 1(\bmod p) .
\end{aligned}
$$

The exponent to which $a$ belongs must be a divisor of $2 r$, but not of $r$, and is therefore even.

If $a$ belongs to the odd exponent $2 k+1(\bmod q)$, then

$$
a^{2 k+1} \equiv 1(\bmod q),
$$

hence $q$ is a divisor of $a^{2 k+1}-1$. Conversely, if $q$ is a divisor of $a^{2 s+1}-1$, then

Presented to the Society, November 20, 1959; received by the editors August 7, 1959. 


$$
a^{28+1} \equiv 1(\bmod q) .
$$

The exponent of $a(\bmod q)$ must be a divisor of $2 s+1$, and is therefore odd.

It follows that each odd prime which is relatively prime to $a$ is either a divisor of an integer of form $a^{r}+1$ or of an integer of form $a^{28+1}-1$.

If, in particular, $a=2$, then the primes for which 2 belongs to an even exponent form the set $A$ of Sierpinski, the other odd primes the set $B$. Now 2 is a quadratic nonresidue for the primes $p$ of form $8 n \pm 3$, hence by Euler's criterion

$$
2^{(p-1) / 2} \equiv-1(\bmod p),
$$

and 2 belongs to an even exponent. Moreover, 2 is a quadratic residue for the primes $q$ of form $8 n+7$, hence

$$
2^{4 n+3} \equiv 1(\bmod q),
$$

and the exponent of 2 is odd. Finally, for $p=8 n+1$ we have

$$
2^{4 n} \equiv 1(\bmod p),
$$

and the exponent to which 2 belongs can be even or odd.

B. M. A. Makowski (see [5]) proved that there are infinitely many primes of form $8 n+1$ which belong to $A$ namely the prime divisors of $2^{2^{m}}+1$. This result follows here at once from Theorem 1 since 2 belongs to an even exponent for all these prime divisors. There exist infinitely many such primes since $2^{2^{m}}+1$ and $2^{2^{k}}+1$ are relatively prime for $m \neq k$. Finally all these prime divisors for $m>1$ are of form $8 n+1$ since the odd prime divisors of the $2^{m+1}$ st cyclotomic polynomial have the form $2^{m+1} z+1$.

This is a special case of the following theorem.

THEOREM 2. Let $p$ be a prime of form $8 n+1$. We set

$$
p-1=2^{e} u \quad(u \text { odd }) .
$$

If 2 is a $2^{2}$ th power residue $\bmod p$, then $p$ belongs to the set $B$, otherwise to $A$.

ProOF. If 2 is a $2^{e}$ th power residue, then by Euler's criterion

$$
2^{(p-1) / 2^{e}} \equiv 2^{u} \equiv 1(\bmod p),
$$

hence $p$ belongs to $B$. Otherwise 2 belongs to an even exponent $\bmod p$, and $p$ is an element of $A$ by Theorem 1 .

We shall use the following theorems on the biquadratic and octavic 
character of 2. (See, for instance, the paper of A. L. Whiteman [7].)

If $p$ is a prime of form $8 n+1$, then 2 is a biquadratic residue mod $p$ if and only if $p$ can be represented as $x^{2}+64 y^{2}$. If $p$ is of form $16 n+1$, then 2 is an octavic residue if and only if $p$ can be represented as $x^{2}+256 y^{2}$. If $p$ is of form $16 n+9$, then 2 is an octavic residue if and only if $p$ can be represented as $x^{2}+64 y^{2}$, but not as $x^{2}+256 y^{2}$.

THEOREM 3. The number 2 is a biquadratic nonresidue for the infinitely many primes which can be represented as

$$
17 x^{2}+64 x y+64 y^{2} .
$$

It is an octavic nonresidue for the infinitely many primes of form $16 n+1$ which can be represented as

$$
65 x^{2}+256 x y+256 y^{2}
$$

and for the infinitely many primes of form $16 n+9$ which can be represented as $x^{2}+256 y^{2}$.

All these primes belong to the set $A$.

Proof. Assume that the prime $p$ can be represented by the positive properly primitive quadratic form

(1) $17 x^{2}+64 x y+64 y^{2}=x^{2}+(4 x+8 y)^{2}=x^{2}+16(x+2 y)^{2}$.

Then $x$ must be odd and $4 x+8 y \equiv 4(\bmod 8)$. Hence in the representation of $p$ as sum of two squares one of the squares is odd and the other divisible by 16 , but not by 64 . Since this representation is unique, $p$ cannot be represented as $x^{2}+64 y^{2}$. Hence 2 is a biquadratic nonresidue $\bmod p$, and consequently a $2^{e}$ th power nonresidue, so that $p$ belongs to $A$. It was proved by $\mathrm{H}$. Weber [6] that every positive properly primitive quadratic form represents infinitely many primes. (See also E. Schering [4], P. Bernays [1], W. E. Briggs [2].) Therefore infinitely many primes are represented by (1) and all of them belong to $A$.

Suppose that $p$ is a prime of form $16 n+1$ and can be represented by the form

(2) $65 x^{2}+256 x y+256 y^{2}=x^{2}+(8 x+16 y)^{2}=x^{2}+64(x+2 y)^{2}$.

Then $p$ is a biquadratic residue, but an octavic nonresidue since it is representable as $x^{2}+64 y^{2}$ but not as $x^{2}+256 y^{2}$ because $x+2 y$ is odd. It was proved by A. Meyer [3] that any positive properly primitive quadratic form represents infinitely many primes which belong to a given linear form if at least one such prime exists. Since the prime 577 
is represented by the quadratic form (2) for $x=y=1$ and is of form $16 n+1$, infinitely many primes of form $16 n+1$ are represented by (2) and all of them belong to $A$.

Suppose that $p$ can be represented as $x^{2}+256 y^{2}$ and is of form $16 n+9$. Since $p=281=5^{2}+256$ is such a prime, infinitely many such primes exist. They belong to $A$ since 2 is an octavic nonresidue for each of them.

THEOREM 4. The number 2 is an octavic residue for every prime of form $16 n+9$ which can be represented as $65 x^{2}+256 x y+256 y^{2}$. All these infinitely many primes belong to the set $B$.

Proof. Let $q$ be such a prime. It follows from (2) that 2 is an octavic residue $\bmod q$. Hence $q$ belongs to the set $B$ by Theorem 2 . Since 73 is of form $16 n+9$ and represented by (2) for $x=3, y=-1$, it follows from the theorem of Meyer that there exist infinitely many such primes $q$. This proves the theorem.

\section{BIBLIOGRAPHY}

1. Paul Bernays, Über die Darstellung von positiven, ganzen Zahlen durch die primitiven, binären quadratischen Formen einer nicht-quadratischen Diskriminante, Dissertation, Göttingen, 1912.

2. W. E. Briggs, An elementary proof of a theorem about the representation of primes by quadratic forms, Canad. J. Math. vol. 6 (1954) pp. 353-363.

3. Arnold Meyer, Über einen Satz von Dirichlet, J. Reine Angew. Math. vol. 103 (1888) pp. 98-117.

4. Ernst Schering, Beweis des Dirichletschen Satzes, dass durch jede eigentlich primitive quadratische Form unendlich viele Primzahlen dargestellt werden, Gesammelte Mathematische Werke vol. 2 (1856) pp. 357-365.

5. Waclaw Sierpinski, Sur une décomposition des nombres premiers en deux classes, Collect. Math. vol. 10 (1958) pp. 81-83.

6. Heinrich Weber, Beweis des Satzes, dass jede eigentlich primitive quadratische Form unendlich viele Primzahlen darzustellen fähig ist, Math. Ann. vol. 20 (1882) pp. 301-329.

7. A. L. Whiteman, The sixteenth power residue character of 2, Canad. J. Math. vol. 6 (1954) pp. 364-373.

University of North Carolina 